\title{
8 Indications for Further Research
}

In terms of providing directions for further research, a single case study is always inviting. Additional studies build the empirical basis on the object of enquiry or context in question, in this case, the SC and its use as a method for change agents. It would for example be interesting to see whether findings from the case of this research project also occur in other contexts or by using different methods for data generation and analysis; for example surveys and quantitative tools. To apply and further develop the proposed intervention process could be the objective of a subsequent action research project.

In the view of the author however, the conceptual model that this work proposed and explored analytically in section 6 is the more significant platform for further research; indeed it is intended to be an invitation for it. The model itself may be questioned, tested, expanded, modified or further validated. Likewise, it is an offer to structure a future debate about the SC and provide direction for venturing future research initiatives. While the approach of this work is broad rather than deep, to consider and explore in isolation and more detail individual modules of the model or individual links between these can further the understanding of how the method operates and functions. It would for example be interesting to consider yet other areas of research that individual elements of the model may be linked to - and whether this provides a basis for collaborative projects with researchers from these fields. Likewise to ascertain empirically the differences between the reflective processes in Phase 1 and Phase 3 of the model and which specific type of reflection of reflective capability the method requires in each, may be a fruitful route for exploration. 